\title{
Papers
}

\section{A questionnaire-based survey of errors in diagnostic histopathology throughout the United Kingdom}

\author{
P N Furness, I Lauder
}

\begin{abstract}
Aims-To obtain a crude estimate of the rate at which consultant histopathologists become aware of errors in their work, and to gather information about the circumstances under which errors are made. Methods-A postal questionnaire was sent to all consultant histopathologists (1021) in the United Kingdom.

Results-There was a $19.8 \%$ response to the questionnaire (202 returned). 119 pathologists reported 244 errors, 82 from within the preceding year. $42 \%$ of these errors had affected patient management. Pathologists usually blamed human error or excessive workload; however, data analysis did not reveal an excess of responses from laboratories with heavy workloads. A disproportionately large proportion of errors appear to be made in samples from the lymphoreticular system and by locum consultant pathologists. Conclusions-This study undoubtedly suffered from reporting bias, but the figures suggest that a typical pathologist probably becomes aware of having made a serious diagnostic error approximately once per year. A larger, more systematic study of the circumstances under which errors are likely to occur appears to be justified but would require significant resources because of the large sample size required.
\end{abstract}

(F Clin Pathol 1997;50:457-460)

Keywords: histopathology; audit; error; diagnosis

Department of

Pathology, University of Leicester, Leicester Royal Infirmary, PO

Box 65, Leicester LE2

7LX, United Kingdom

P N Furness

I Lauder

Correspondence to:

Dr Furness.

Accepted for publication 10 March 1997
Histopathologists, like all humans, occasionally make mistakes. This fact has recently been widely recognised through the publicity surrounding a small number of high profile cases. The public and political reaction has been that these are exceptional, and usually a consequence of a pathologist's ill health, but there remain long term doubts about the quality of the overall histopathology diagnostic service.

Retrospective studies of error rates have produced widely different results; one quite large study found no "serious" errors, ' but others have suggested that $0.26 \%^{2}$ or even up to $1.2 \%{ }^{3}$ of histopathological reports are likely to contain a clinically significant error. A review of cases reviewed at clinicopathological case conferences-admittedly a selected groupshowed an altered diagnosis in $9 \%$ of cases, with major management changes resulting in $3.8 \%{ }^{4}$ Obviously there is considerable uncertainty about what errors are considered to be significant in such studies. Some have considered only errors in the final diagnosis, ${ }^{2}$ others have considered the possibility of errors throughout the process from taking the specimen to receipt of the report. ${ }^{56}$ These studies have all been carried out in single specialist institutions that may not be representative of the wider picture. They have not addressed in any depth the circumstances under which the errors were made.

A recent United Kingdom Department of Health inquiry documented a large number of errors made by a single locum histopathologist. ${ }^{7}$ The inquiry concluded that the number of errors made was probably excessive, but was unable to be certain because of the absence of hard data on what is a "normal" error rate. One of the recommendations of this report was that research to resolve this uncertainty should be carried out. Such data would be invaluable to any pathologist accused of making an unacceptable number of errors, a situation which could occur after just one incident if managers assume that histopathology departments should never make mistakes. Information on diagnostic errors would also be helpful in planning histopathology services; to reduce errors, we need to know under what circumstances errors are more likely to occur.

A definitive study to resolve these questions would need to cover a large number of institutions, and would probably require that expert outside assessors examine a large random sample of cases within each laboratory, attempting to find errors. Sophisticated data analysis would be required. This would be very expensive and so far funding for such a study has not been forthcoming. We therefore decided to attempt a preliminary study by sending a questionnaire to all consultant histopathologists in 


\section{Survey of errors in histopathology diagnosis}

Please do not write anything on this form which would permit identification of yourself, the specimen, the patient or the hospital.

When complete, please return to The Postgraduate Dean's Secretary, Robert Kilpatrick Clinical Sciences Building, Leicester Royal Infirmary, in the enclosed reply-paid envelope. The postmark and any identifyin details will be stripped off before the form is passed to us for analysis.

\section{Departmental statistics}

This information is essential if we are to relate the occurrence of mistakes to the circumstances under which they are made.

Please include only one copy of this side of the form, even if several errors are reported.

Workload for 1994 (numbers of requests):
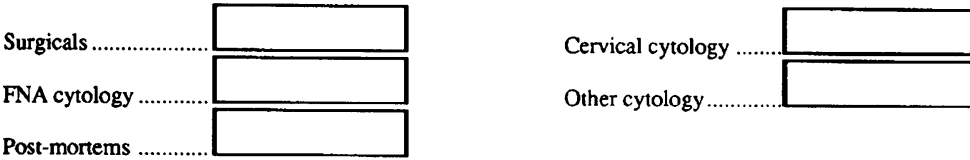

Staffing:

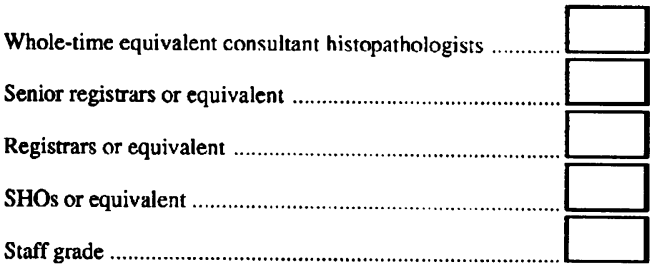

Is yours a teaching hospital? Yes/No (Please delete as appropriate)

Any special advantages or disadvantages not covered above?

Figure 1 The questionnaire used to obtain information about the department where a mistake had occurred.

\section{Materials and methods}

A questionnaire (figs 1 and 2) was sent to each member of the Royal College of Pathologists who appeared, from the information available in the College handbook, to have histopathology as their main speciality, to be resident in the UK, and not retired.

A covering letter explained our request, our aims, and our belief that the information to be gained was potentially of value to the profession. Recognising that there might be reluctance to divulge such sensitive information, we emphasised that we did not wish to receive any information that might allow the sender to be recognised. The responses were to be posted not to us, but to the secretary of the Postgraduate Dean in Leicester, who had agreed to remove the outer envelopes and any postmark before passing the questionnaires to us for analysis.

Figure 1 relates to the institution, and was to be filled out once by each respondent. The form shown in figure 2 describes the mistake, was to be photocopied if necessary, and a separate copy filled out for each error of which the submitting pathologist was aware. The questions illustrate our attempts to determine the circumstances under which each error was made.

We emphasised that if a pathologist could remember no errors, we would welcome a return to that effect, to reduce the inevitable bias which would result from an incomplete return of the questionnaire.

The results of the questionnaires were entered by a secretary into a database written for the purpose using Omnis 7 (Blyth Software, Berkshire, UK); the subsequent analysis involved interrogation of this database, and the original paper questionnaires were destroyed. Pathologists. We recognised from the outset that this would inevitably be an incomplete and potentially biased assessment, providing anecdotal rather than definitive evidence. We nevertheless thought that it could provide information that is currently unavailable, and might assist in refining the design of a subsequent definitive study.

Table 1 Free text comments on causes of errors

Problems with locums

Colleague on leave, no locum

Colleague not sharing workload

Strain due to management duties

Split site working

Single handed

Staffing levels, MLSOs and medical

Demands of very specialised departments

Problems with computer system

Academics not doing NHS work

Academics doing NHS work

Research and teaching workload

Inadequate clinical information

Inappropriate specials

Ignored $x$ rays

Technically poor frozen section

Local specialist away

Muddled at 2:00 am

Domestic crisis, bereavement.

Excessive reliance on previous diagnosis

Inadequate specimen/fixation

The word "no" omitted from report

No explanation, the report bore no relationship to the sections

\section{Results}

A total of 1021 questionnaires were sent and 202 were returned $(19.8 \%)$. Inevitably, the mailshot included some pathologists with no diagnostic pathology workload, such as full time forensic pathologists and some senior registrars ( 25 responses indicated that this was the case). Eight pathologists wrote indicating that they thought the profession was not best served by investigating errors, or that they would not wish to collaborate with any study less perfect than the prohibitively expensive one described above. From the 988 remaining, we received 169 responses $(17.1 \%)$. Of these, 47 indicated no knowledge of any errors in the past five years and 119 pathologists reported a total of 244 errors. Of these $183(75 \%)$ were small diagnostic biopsies; 61 (25\%) from therapeutic resections; $51(21 \%)$ were made in teaching hospitals; $234(96 \%)$ had potential to alter management; 102 (42\%) actually did alter patient management; $173(71 \%)$ represented a benign/malignant error.

A breakdown of some of the features is given in figures 3 to 7 . A precis of some of the free text comments is given in table 1 . 
Errors of potential clinical significance in the last five years

Please complete one copy of this side of the form for each error.

Approximate date when the error occurred :

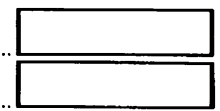

Approximate date when the error was discovered :

Was the error:

Made by you?

Made a locum consultant pathologist? .. $\square$ *

Made by a trainee pathologist? ............ $\square$

Made by technical staff? .......................

Made by postal / portering staff? .........

Made by another consultant pathologist?.. $\square$ Made by a staff grade pathologist?...

Made by secretarial staff? D

Made by clinical staff? .

(Please tick appropriate box(es))

* This request is being sent to all consultant histopathologists currently in post. If the error was made by a pathologist other than yourself, please check that only one form is being returned.

Did this error have potential to alter patient management? ............. Yes/No (Please delete as appropriate)

Did this error actually alter patient management? ........................... Yes $/$ No iPleave delete as appropriate)

Has this error resulted in litigation?

Yes /No i Please delete as appropriate,

If Yes, please give any available details of outcome, especially estimated / actual cost.

In your view, is this likely to result in litigation? ......................... Yes/No (Please delete as appropriate)
In your view, would litigation be successful? .............................. Yes/No (Please delese as appropriate)
Was this a benign / malignant error? ............................................. Yes/No (Pleave delete as appropriate)

What organ system did this involve?

Was the specimen: A small diagnostic biopsy? $\square \quad$ A therapeutic resection?

(Please tick one)

Which (if any) of the following factors were involved in the cause of this error? (Please tick as many as are Which (if
relevant)

$\begin{aligned} & \text { Human error ............................ } \square \text { Excessive workload............. } \\ & \text { Special studies not requested .... } \text { Poor facilities ............................. } \\ & \text { Ill health .................................. }\end{aligned}$ Other factors contributing to this error:

Figure 2 The questionnaire used to obtain information about each mistake.

\section{Discussion}

We fully recognise that this information is largely anecdotal. Our response rate is respectable by the standards of most postal questionnaires, but we do not know whether the large number of pathologists who did not reply did so out of apathy, because they were reluctant to report mistakes, or because they were aware of none. We think the last possibility unlikely. We do not know whether pathologists with heavy workloads had less time to complete our questionnaire. Our study would omit all mistakes that were not detected and, as shown in figure 3, many which have been forgotten. Despite these limitations, in many respects this is the best, or the only, information available.

Clearly we cannot infer the rate at which errors are made with any accuracy. As an approximation we can say that, for the population of pathologists who responded, it is normal for a pathologist to become aware of having made a diagnostic error a little under once every year; approximately once every 5000 specimens. Of these, approximately half have significant clinical impact. If this is a representative sample, the potential damage being caused is probably sufficient to justify a

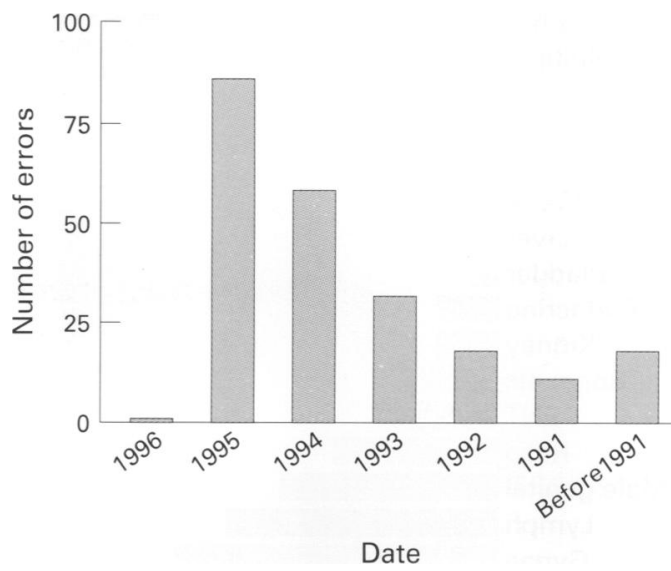

Figure 3 The number of errors reported, broken down by year detected. The questionnaires were posted early in 1996.

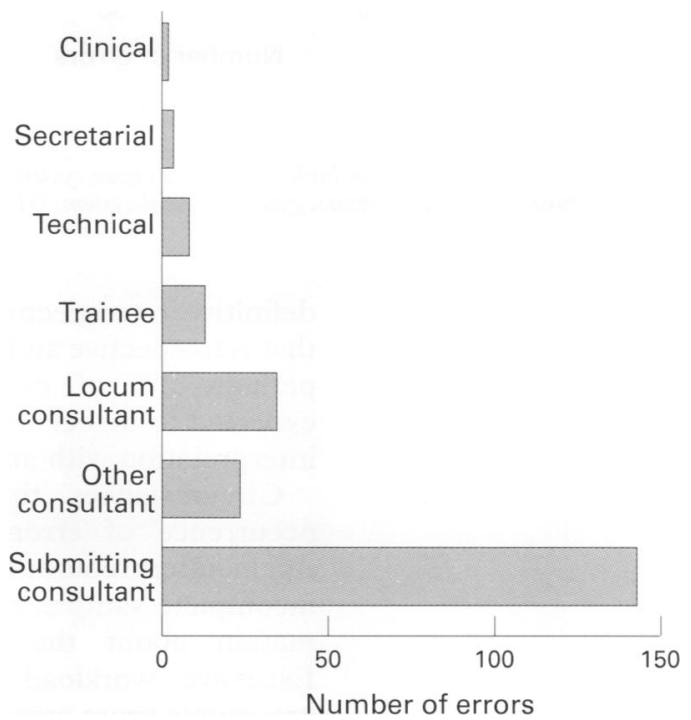

Figure 4 The person principally responsible for having made the reported errors.

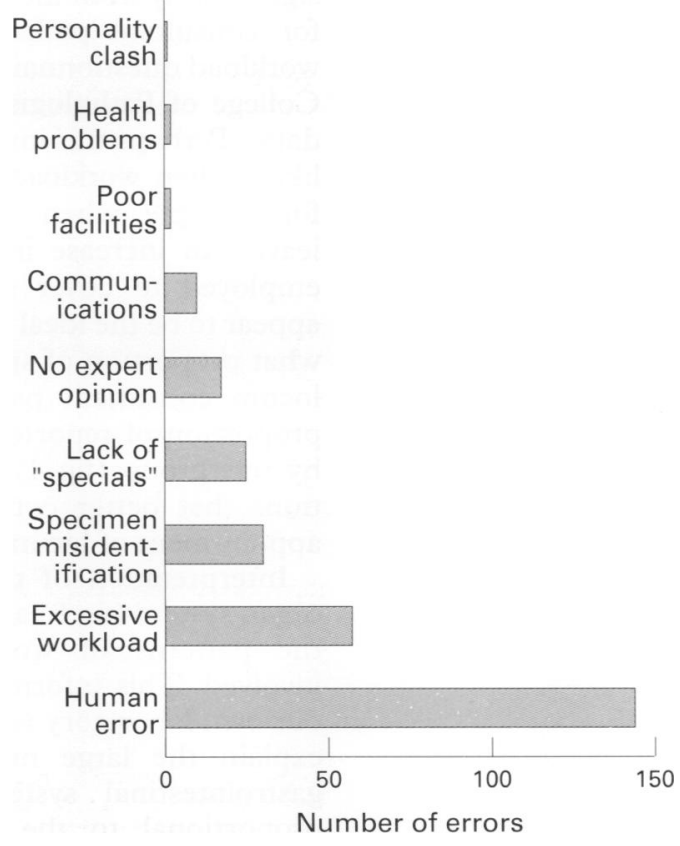

Figure 5 Factors which, in the opinion of the submitting consultant, had contributed to the production of an error. 


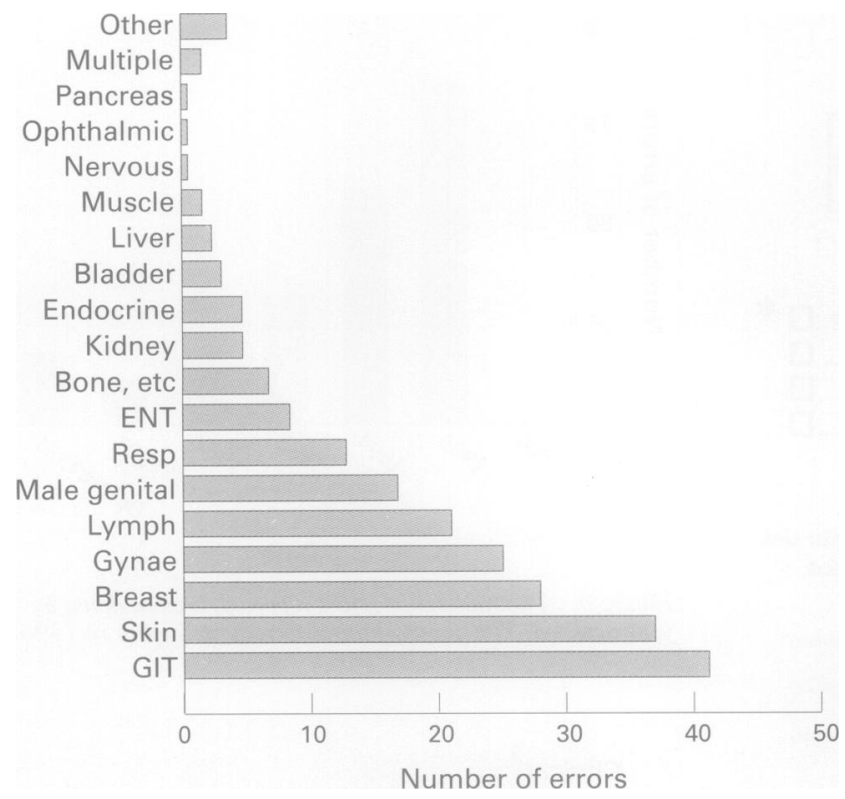

Figure 6 Numbers of errors broken down by organ system. ENT, ear, nose, and throat; Resp, respiratory system; Gynae, gynaecological system; GIT, gastrointestinal tract.

definitive retrospective study. It also suggests that retrospective audits of a random sample of perhaps $2 \%$ of cases reported cannot be expected to detect serious errors of diagnostic interpretation with any speed or reliability.

Circumstances that might promote the occurrence of errors have been suggested. Again, interpretation is limited, not only by our incomplete sample, but also by limited information about the laboratories concerned. Excessive workload might be expected to precipitate more errors, and this was the single most commonly quoted feature contributing to individual errors. However, if we consider the distribution of workloads in the laboratories (fig 7), the distribution does not differ significantly from the national workload figures for consultant pathologists provided by the workload questionnaire circulated by the Royal College of Pathologists in 1993 (unpublished data). Perhaps this implies that errors are more likely when workloads temporarily increasefor example, when colleagues are on annual leave. An increase in the number of locums employed to cover such absences does not appear to be the ideal solution. We do not know what proportion of specimens are reported by locum consultant pathologists, but the high proportion of reported errors that were made by this group (fig 4) supports recent suggestions that better controls are needed in the appointment of locums. ${ }^{7}$

Interpretation of the number of errors by organ system (fig 6) also requires knowledge of the patterns of work of the departments involved. This information is not available. If our own laboratory is representative, we might explain the large number of errors in the gastrointestinal system and skin as being proportional to the large number of such

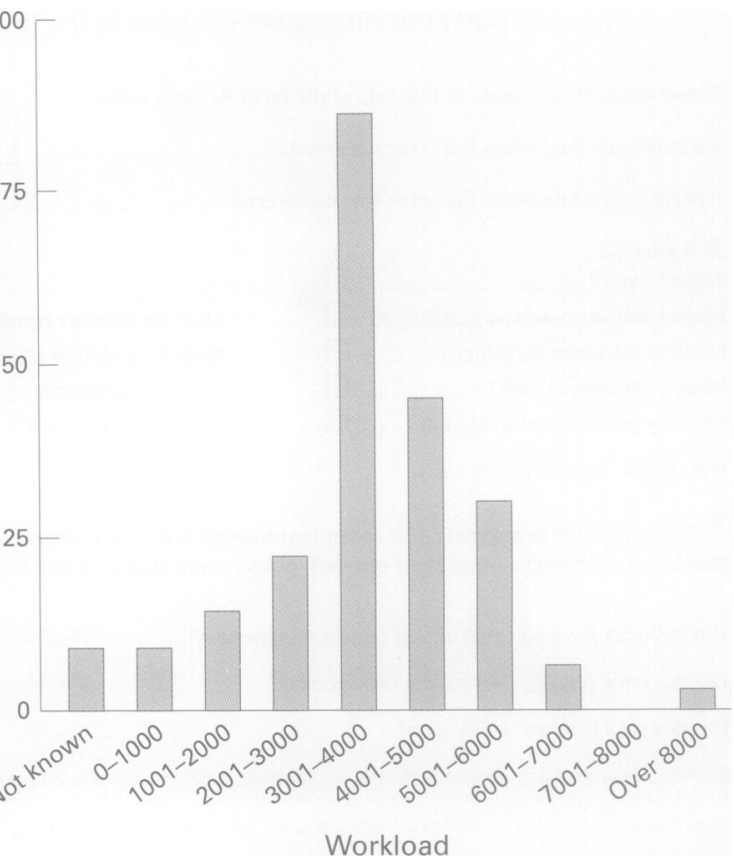

Figure $7 \quad$ Numbers of errors broken down by the surgical pathology workload of the departments in which they occured (expressed as cases per whole-time equilavent consultant per year).

specimens we see from those systems. The number of errors in breast pathology is perhaps out of proportion to the number of specimens, but the clearest danger area is the lymphoreticular system, where the number of errors reported is totally out of proportion to the number of biopsies we handle. This has implications in terms of the availability (or otherwise) of specialist second opinions in some of the more difficult areas of diagnostic histopathology.

In summary, we have demonstrated that it is not rare for a diagnostic histopathologist to make an error. The potential impact of these errors on patient care is considerable, and we suggest that our results justify the expense of a carefully constructed retrospective study of the frequency of errors, and more importantly, the circumstances under which they occur.

We are grateful for the skilled data entry and secretaria assistance provided by Mrs Wendy Pitts.

Cree IA, Guthrie W, Anderson JM, Holley MP, Hopwood $D$, Sanders DSA, et al. Departmental audit in histopathol ogy. Pathol Res Pract 1993;189:453-57.

2 Safrin RE, Bark CJ. Surgical pathology signout-routine review of every case by a 2 nd pathologist. Am $\mathcal{F}$ Surg Patho 1993;17:1190-2.

3 Lind AC, Bewtra C, Healy JC, Sims KL. Prospective peerreview in surgical pathology. Am $\mathcal{f}$ Clin Pathol 1995;104 $560-6$

4 McBroom HM, Ramsay AD. The clinicopathological meeting - a means of auditing diagnostic performance. $A m$ f Surg Pathol 1993;17:75-80.

5 Ramsay A. Locally organised medical audit in histopathology. $¥$ Clin Pathol 1991;44:353-7.

6 Ramsay AD, Gallagher PJ. Local audit of surgical pathology-18 months experience of peer-review based pathology-18 months experience of peer-review based quality assessment in an Englis

7 Malley R, O'Brien J, Anderson M, Davies D. Final report of the review into the appointment of Dr SW Kiberu as a locum consultant histopathologist at Grantham and Kesteven General Hospital and at Bassetlaw Hospital and Community Services NHS Trust. NHS Executive (Trent) Department of Health, UK Govermnent, 1996. 